\title{
Intertrial Variability in the Premotor Cortex Accounts for Individual Differences in Peripersonal Space
}

\author{
DiDrancesca Ferri, ${ }^{1}$ Marcello Costantini, ${ }^{1,2}$ Zirui Huang, ${ }^{1}$ Mauro Gianni Perrucci, ${ }^{2}$ Antonio Ferretti, ${ }^{2}$ \\ Gian Luca Romani, ${ }^{2}$ and Georg Northoff ${ }^{1}$ \\ ${ }^{1}$ Institute of Mental Health Research, University of Ottawa, Ottawa, Ontario K1Z 7K4, Canada, and ${ }^{2}$ Department of Neuroscience, Imaging, and Clinical \\ Science, G. d'Annunzio University of Chieti and Institute for Advanced Biomedical Technologies, Chieti 66100, Italy
}

We live in a dynamic environment, constantly confronted with approaching objects that we may either avoid or be forced to address. A multisensory and sensorimotor interface, the peripersonal space (PPS), mediates every physical interaction between our body and the environment. Behavioral investigations show high variability in the extension of PPS across individuals, but there is a lack of evidence on the neural underpinnings of these large individual differences. Here, we used approaching auditory stimuli and fMRI to capture the individual boundary of PPS and examine its neural underpinnings. Precisely, we tested the hypothesis that intertrial variability (ITV) in brain regions coding PPS predicts individual differences of its boundary at the behavioral level. Selectively in the premotor cortex, we found that ITV, rather than trial-averaged amplitude, of BOLD responses to far rather than near dynamic stimuli predicts the individual extension of PPS. Our results provide the first empirical support for the relevance of ITV of brain responses for individual differences in human behavior.

Key words: fMRI; interindividual differences; intertrial variability of brain response; looming sounds; peripersonal space

Significance Statement

Peripersonal space (PPS) is a multisensory and sensorimotor interface mediating every physical interaction between the body and the environment. A major characteristic of the boundary of PPS in humans is the extremely high variability of its location across individuals. We show that interindividual differences in the extension of the PPS are predicted by variability of BOLD responses in the premotor cortex to far stimuli approaching our body. Our results provide the first empirical support to the relevance of variability of evoked responses for human behavior and its variance across individuals.

\section{Introduction}

In everyday life, we are immersed in a dynamic space. As we walk down the street, we are approached by dogs, cars, bicycles, and various objects that we may have to either avoid or address. In the long struggle for survival, animals and humans have learned that a dynamic object that approaches one's body is far more of a threat than a static object. Therefore, they developed defensive behavior (Schiff, 1965; Fotowat and Gabbiani, 2011) to protect their body surface from attack or collision. When, though, do approaching stimuli begin to elicit motor responses from us?

\footnotetext{
Received April 29, 2015; revised 0ct. 5, 2015; accepted 0ct. 13, 2015.

Author contributions: F.F., M.C., and G.N. designed research; F.F., M.G.P., and A.F. performed research; F.F. and Z.H. analyzed data; F.F., M.C., Z.H., G.L.R., and G.N. wrote the paper.

We thank Giulio Di Cosmo for help in collecting behavioral data and Annamarie Wolff and Yuliya Nikolova for comments on the manuscript.

The authors declare no competing financial interests.

Correspondence should be addressed to Francesca Ferri, Institute of Mental Health Research, University of 0ttawa, Ottawa, Ontario K1Z7K4, Canada. E-mail: fraferri78@gmail.com.

DOI:10.1523/JNEUROSCI.1696-15.2015

Copyright $\odot 2015$ the authors $\quad 0270-6474 / 15 / 3516328-12 \$ 15.00 / 0$
}

Behavioral investigations have consistently shown that stimuli in one sensory modality augment the processing of stimuli in another, especially when the stimuli are perceived as potentially interacting with our body (Graziano and Cooke, 2006), that is, as soon as they cross the boundary of what has been described as "peripersonal space" (PPS) (Rizzolatti et al., 1997). PPS is a multisensory and sensorimotor interface mediating every physical interaction between the body and the environment (Làdavas and Serino, 2008). It represents the portion of the space where sensory signals from different modalities can be integrated (Brozzoli et al., 2014) and trigger motor responses (Maravita et al., 2003). A major characteristic of the boundary of PPS in humans is the extremely high variability of its location across individuals (Longo and Lourenco, 2007; Lourenco et al., 2011; Sambo and Iannetti, 2013; Taffou and Viaud-Delmon, 2014). The neural origins of the large individual differences in the location of PPS boundary currently remain unclear.

Another key characteristic of PPS is that its sensorimotor representation is plastic and dynamic (Cléry et al., 2015). Interestingly, computational, modeling and neurophysiological studies 
(Churchland et al., 2006; Rokni et al., 2007; Faisal et al., 2008) suggest that sensorimotor plasticity and dynamic representations are enabled by neural variability. For instance, the increase of intertrial variability (ITV) in monkey motor cortices has been shown to enable dynamic adaptation to new environments (Mandelblat-Cerf et al., 2009). Drawing from the evidence of a pivotal role of neural variability in dynamic representations, we hypothesized a relationship between ITV of BOLD responses to approaching stimuli and the individual location of the PPS boundary. Specifically, higher ITV levels should characterize individuals with a narrow PPS boundary because they afford many possible sensorimotor states to explore. However, whereas variability, especially ITV, has been validated in animal studies at the cellular level (Carandini, 2004; Scaglione et al., 2011; Marcos et al., 2013), it has so far been used rarely in human imaging studies at the regional level $(\mathrm{He}, 2013)$. In addition, evidence is still lacking on elucidating the contribution of regional ITV dynamics for human behavior.

In this context, the aim of this study was to identify neural predictors of individual differences (Kanai and Rees, 2011) of the PPS boundary using fMRI. Specifically, we test the hypothesis that ITV levels in brain regions coding PPS predict individual differences of PPS representation at the behavioral level. But which are the candidate brain regions where ITV changes could predict individual PPS boundary? Neurophysiological and neuroimaging investigations (Rizzolatti et al., 1981; Fogassi et al., 1996; Bremmer et al., 2001; Brozzoli et al., 2011, 2014) have shown that the premotor cortex (PM) plays a crucial role in motor mapping of sensory events occurring near the body, especially in humans (Avenanti et al., 2012).

According to the sensorimotor nature of PPS, its neural underpinnings are more effectively recruited by approaching over receding or static stimuli (Colby et al., 1993; Fogassi et al., 1996; Graziano et al., 1997). Surprisingly, all neuroimaging studies investigating PPS in humans have used visual stimuli presented at fixed locations near or far from the body (Makin et al., 2007; Brozzoli et al., 2011, 2013). Therefore, in the present study, we used a recently proposed audio-tactile interaction task (Canzoneri et al., 2012) using looming sounds to assess PPS boundaries dynamically along a spatial continuum.

\section{Materials and Methods Participants}

Thirty-eight healthy volunteers ( 12 females, mean age 21.8 years, range $20-31)$ participated in the behavioral audio-tactile interaction task (PPS) and in the fMRI audio-tactile interaction task (PPS). Three separate groups of healthy volunteers ( $n=20$ in each group) were recruited for the following behavioral control studies: (1) reproducibility of the $\mathrm{CP}$ across sessions (10 females, mean age 22.5 years, range $21-31$ ); (2) reproducibility of the $\mathrm{CP}$ across tasks ( 12 females, mean age 22.0 years, range 20-29); (3) sound localization task (11 females, mean age 22.1 years, range 20-31). All the participants were right handed and took part in the studies after providing written informed consent. The experimental protocol was approved by the University G. D'Annunzio of Chieti institutional ethics committee.

\section{Stimuli}

Auditory stimuli, presented during the behavioral sessions and the fMRI session, were samples of pink noise (or $1 / \mathrm{f}$ noise) of $3100 \mathrm{~ms}$ duration with flat or increasing (looming) intensity levels. The sounds were sampled at $44.1 \mathrm{kHz}$. Sound intensity was manipulated using Soundforge 4.5 software (Sonic Foundry) so that "looming sounds" had exponentially rising acoustic intensity from 55 to $70 \mathrm{~dB}$, whereas "flat sounds" had constant $62.5 \mathrm{~dB}$ acoustic intensity. During the behavioral sessions, au- ditory stimuli were presented by two loudspeakers (see below), whereas during the fMRI session, the same stimuli were delivered by headphones (specifically designed for fMRI and connected to a NordicNeuroLab audio system). In a separate control study, we checked that perceived stimulus distance was not affected by the different experimental setup during the fMRI session compared with the behavioral sessions (see "Sound localization task" section).

Tactile stimuli, presented during the behavioral sessions and fMRI session, were delivered by means of constant-current electrical stimulators (DS7A; Digitimer) via pairs of neurological electrodes placed on the hairy surface of the index fingers. The electrical stimulus was a single, constant voltage, rectangular monophasic pulse. At the beginning of each session, the intensity of the tactile stimulus was set to be clearly above thresholds individually for each participant (Canzoneri et al., 2012). Intensity of the stimulator was set at the minimum value and then progressively increased until the participant reported to clearly perceive the stimulation. Next, the participant was presented with a sequence of 10 stimuli, intermingled with five catch trials in which no stimulation was presented. He/she was asked to report when he/she felt the tactile stimulus. If the participant did not perform $100 \%$ correctly (i.e., if he/she failed to respond to some stimuli or gave false positives to the catch trials), the intensity was further increased by a $5 \mathrm{~mA}$ step and the procedure was repeated. Intensity for the tested participants ranged between 60 and $90 \mathrm{~mA}$. Stimulus duration was equal to $100 \mu \mathrm{s}$.

The presentation of auditory and tactile stimuli, as well as the recording of participants' responses, was controlled by custom software implemented in MATLAB (The MathWorks).

\section{Behavioral sessions}

Behavioral audio-tactile interaction task (PPS)

Apparatus and procedure. During the experiment, participants were blindfolded and comfortably seated beside a table with their right arm resting palm down. The audiotactile apparatus, which was mounted on the table, consisted of two loudspeakers, one placed near to the participants' right hand and the other at a distance of $100 \mathrm{~cm}$ from the near loudspeaker (i.e., far from the participant) and a constant-current electrical stimulator controlling a pair of neurological electrodes attached on the participant's right index finger. During each trial, either a looming or a flat sound was presented. Along with the auditory stimulation, in the $60 \%$ of trials, participants were also presented with a tactile stimulus. The remaining trials $(40 \%$ of total) were catch trials with auditory stimulation only (either looming or flat sounds).

The tactile stimulus was delivered at varying temporal delays from the onset of the auditory stimulus. Five different temporal delays were used: T1, $300 \mathrm{~ms}$; T2, $800 \mathrm{~ms}$; T3, $1500 \mathrm{~ms}$; T4, $2200 \mathrm{~ms}$; and T5, $2700 \mathrm{~ms}$ (Fig. 1a). Each trial was followed by an intertrial interval of $1000 \mathrm{~ms}$. Each participant was presented with a random combination of 18 target stimuli for each temporal delay for the looming and flat sounds randomly intermingled with the catch trials. Trials were equally divided into three blocks.

Participants were asked to respond as fast as possible to the tactile target, when present, by pressing a button on a response box (Cedrus RB-834) with their left index finger, trying to ignore the auditory stimulus.

Data analysis. To investigate the specific impact of the perceived position of approaching sounds compared with flat sounds on participants' responses, mean reaction times (RT)s to tactile targets were calculated for every temporal delay separately for looming and flat sounds. Data on all participants were entered in an ANOVA with factors sound (looming, flat) and temporal delay (T1, T2, T3, T4, T5). Significant effects found in the ANOVA $(\alpha$ level $=0.05)$ were followed by Bonferroni-corrected post hoc analyses. Then, to estimate the individual boundary of PPS representation, mean RTs to the tactile targets at the different temporal delays (T1-T5) were fitted to a sigmoidal function (Canzoneri et al., 2012; Teneggi et al., 2013; Ferri et al., 2015) as follows:

$$
y(x)=\frac{y_{\min }+y_{\max } \cdot e^{\left(x-x_{e}\right) / b}}{1+e^{\left(x-x_{e}\right) / b}}
$$



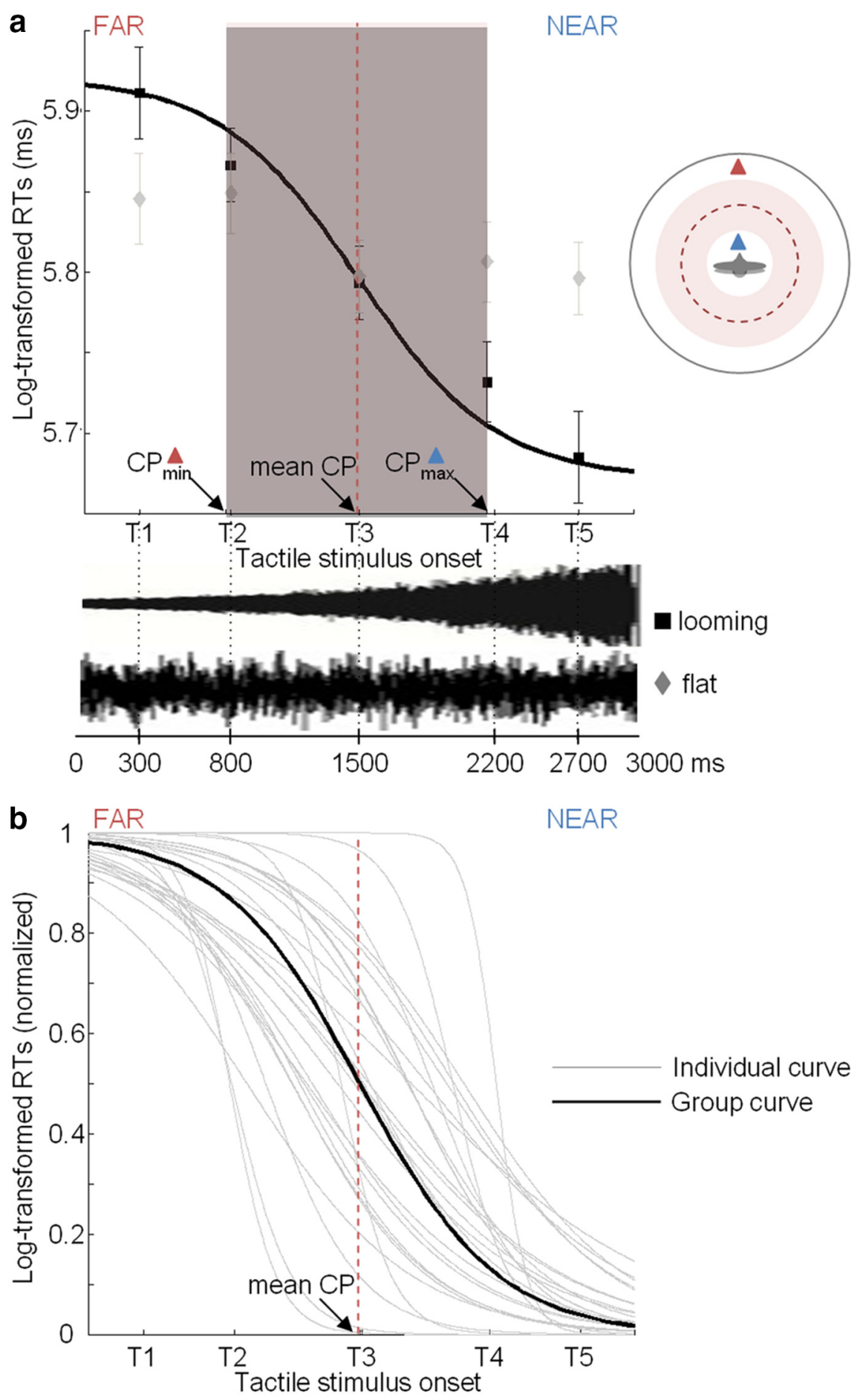

Figure 1. Behavioral differences of PPS boundary across participants. Participants were asked to respond as fast as possible to a tactile target administered on their right index finger, along with a task-irrelevant auditory stimulus. $\boldsymbol{a}$, Top, Plots of mean log-transformed RTs to the tactile target concurrent with either looming or flat sounds. Data averaged across 28 participants are reported. Vertical bars indicate SEs. Continuous line represents the best fitting sigmoidal function. CP identifies the location of the PPS boundary and corresponds to the critical distance at which sound affected the participant's tactile RTs. The shaded region indicates the total range of (CP values across participants, from the minimum (CPmin $=774 \mathrm{~ms})$ to the maximum (CPmax $=2241$ $\mathrm{ms}$ ) value and the dashed red vertical line indicates the group (P (mean (P). Right, Top view of participants' PPS boundaries. Bottom, Auditory stimuli. Looming sounds increased their intensity in time giving the impression of an approaching object. Flat sounds had constant auditory intensity. They allowed controlling for the impact of auditory intensity changes on audio-tactile interaction. Tactile stimuli were delivered at five different temporal delays (T1-T5): T1, 300 ms after the sound onset; T2, $800 \mathrm{~ms}$; T3, $1500 \mathrm{~ms} ; \mathrm{T4}, 2200 \mathrm{~ms}$; and T5, $2700 \mathrm{~ms}$. Therefore, they occurred when the approaching sound source was perceived either close to the body (higher temporal delays) or far from the body (lower temporal delays). Each trial was followed by an intertria interval of 1000 ms. $\boldsymbol{b}$, Individual sigmoid fits obtained from log-transformed RTs. Log transformation was applied
Where $x$ represents the independent variable (timing of touch delivery in milliseconds); $y$ the dependent variable (RT); $y_{\text {min }}$ and $y_{\text {max }}$ the lower and upper saturation levels of the sigmoid, respectively; $x_{c}$ the value of the abscissa at the $\mathrm{CP}$ of the sigmoid (value of $x$ at which $\left.y=\left(y_{\min }+y_{\max }\right) / 2\right)$; and $b$ establishes the slope of the sigmoid at the CP. We first checked that, for looming but not for flat sounds, this sigmoid function provided the best description of the relationship between RTs and timing of tactile stimulus delivery compared with a linear function. The linear function was described by the following equation: $y(x)=y_{0}+k x$, where $x$ and $y$ have the same meaning as above, $y_{0}$ represents the intercept at $x=0$, and $k$ is the slope of the linear function. We compared the root mean square error (RMSE), an index of goodness of fit, between the two models by means of a paired $t$ test analysis. Because the estimated parameters for a linear model are two (i.e., the intercept, $y_{0}$, and the slope, $k$ ), to make the comparison possible, we similarly limited the estimated parameters for the sigmoid model to two (i.e., the central position, $x_{\mathrm{c}}$, and the slope at the CP, $b$ ). To this end, for each participant, values of the parameters $y_{\min }$ and $y_{\max }$ were assigned a priori equal to the minimum and maximum values of individual dataset. For each participant, we then took $x_{\mathrm{c}}$, hereafter referred to as the $\mathrm{CP}$ of the curve, as an estimation of the individual boundary of PPS representation (Canzoneri et al., 2012; Teneggi et al., 2013; Ferri et al., 2015). To assess the statistical acceptance of individual CPs, we ran $t$ test analyses using OriginLab (http:// www.originlab.com). The null hypothesis for these analyses is that the parameter is equal to zero. For each participant, we calculated the one-tailed probability values (individual CPs can be positive only) of the $t$ test on the parameter of interest $(\alpha$ level $=0.05)$.

Definition of individual $\mathrm{T}_{N E A R}$ and $\mathrm{T}_{F A R}$ delays for the fMRI session

Mean RTs to tactile targets delivered along with looming sounds were also used to define the individual near $\left(T_{\mathrm{NEAR}}\right)$ and Far $\left(T_{\mathrm{FAR}}\right)$ conditions for the fMRI session. For each participant, $T_{\text {NEAR }}$ was assigned to the temporal delay associated with their fastest and less variable mean RTs, whereas $T_{\mathrm{FAR}}$ was assigned to the temporal delay associated with their slowest and less variable mean RTs. This allowed presenting stimuli having comparable subjective effectiveness across participants as revealed by both the magnitude and the consistency of their facilitating effect on individual RTs. Therefore, during the fMRI session, each participant received the tactile stimulus when they clearly and consistently perceived the approaching sound source as being far from their body at $T_{\mathrm{FAR}}$ and close to ticipants showing a statistically acceptable (P estimate, as computed by OriginLab, are included ( $n=28 / 36)$. 


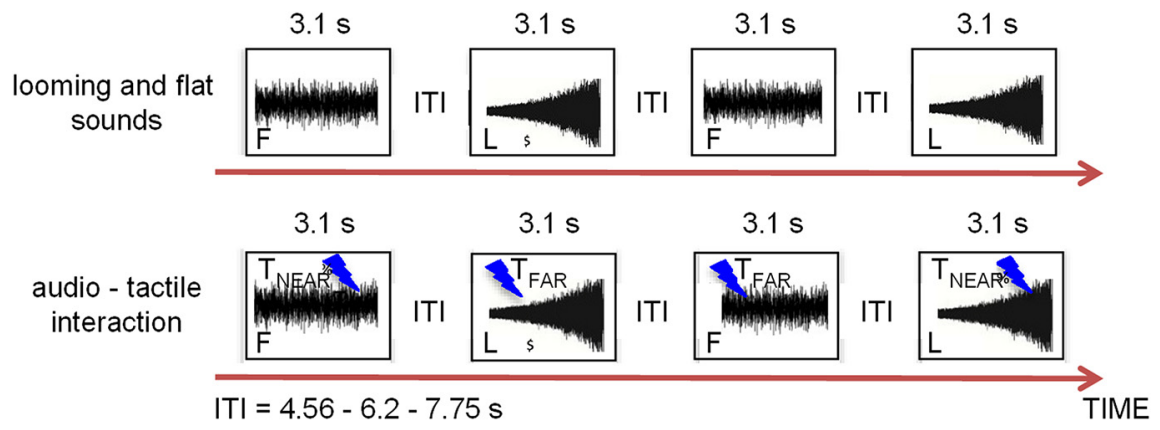

Figure 2. Study design. We recorded BOLD signal time series during two different tasks. Top, The auditory task aimed at identifying looming-sensitive brain regions, regions producing higher activity in response to looming than flat sounds. Two experimental conditions were presented: looming sound, L, and flat sound, F. Bottom, The PPS task aimed at investigating the neural underpinnings of participants' $P$ PS, regions showing modulation of BOLD responses to audio-tactile interaction due to the perceived distance of looming sounds, compared with flat sounds. Four experimental conditions were presented that resulted from the combination of the two types of sounds ( $($ and $F)$ and the two temporal delays of the tactile stimulus $\left(T_{\text {NEAR }}\right.$ and $\left.T_{\text {FAR }}\right) \cdot T_{\text {NEAR }}$ and $T_{\text {FAR }}$ were individualized for each participant based on their behavioral performance. $T_{\text {NEAR }}$ was assigned to the temporal delay associated with the participant's fastest mean RTs, whereas $T_{\mathrm{FAR}}$ was assigned to the temporal delay associated with the participant's slowest mean RTs. $L T_{\text {NEAR }}$ and $L T_{\text {FAR }}$ conditions allowed testing the effect of looming sounds on audio-tactile interaction occurring at temporal delays that corresponded to the individually perceived near and far space. $F T_{\text {NEAR }}$ and $F T_{\text {FAR }}$ control conditions allowed obtaining BOLD responses to audio-tactile interaction at each temporal delay that were not affected by sound intensity changes. ITI, Intertrial interval.

their body at $T_{\mathrm{NEAR}}$. $T_{\mathrm{NEAR}}$ for the tested participants were as follows: $1500 \mathrm{~ms}$ (4 participants), $2200 \mathrm{~ms}$ (14 participants), or $2700 \mathrm{~ms}(20$ participants) after sound onset, whereas $T_{\mathrm{FAR}}$ were $300 \mathrm{~ms}$ (25 participants) or $800 \mathrm{~ms}$ (13 participants) after sound onset.

\section{Reproducibility of the $\mathrm{CP}$ across} sessions

To demonstrate the reproducibility of individual CPs across sessions, we asked a new group of participants to perform the same audio-tactile interaction task described above twice (see "Behavioral audio-tactile interaction task (PPS)" section). The two experimental sessions were separated by at least 1 week. Individual CPs were computed as described above.

\section{Reproducibility of the CP across tasks}

The adaptation of the behavioral audio-tactile interaction task (PPS) for the fMRI session will require delivering tactile stimuli also on the participants' left hand (see below) rather than only on their right hand, as in the behavioral task (see "Behavioral audio-tactile interaction task (PPS)" section). To rule out the possibility that attention shift during the fMRI session may affect individual PPS boundaries, we ran two behavioral experiments on a new group of participants. The stimuli were the same as those described in the "Behavioral audio-tactile interaction task (PPS)" section. The two experiments were identical except for the catch trials. In fact, in the first experiment, the catch trials $(40 \%$ of total) were with auditory stimulation only (either looming or flat sounds), whereas in the second experiment, the catch trials $(40 \%$ of total) were trials in which a tactile stimulus was delivered on the left index finger paired with either a looming or a flat sound. In the first experiment, participants were instructed to refrain from responding when the tactile stimulus was not delivered, whereas in the second experiment, they were instructed to refrain from responding to the left tactile stimulus. All the participants performed both studies (Experiment 1 and 2) in a randomized order.

\section{Sound localization task}

Because we defined near $\left(T_{\mathrm{NEAR}}\right)$ and far $\left(T_{\mathrm{FAR}}\right)$ conditions for the fMRI study according to participants' behavioral performance (see "Definition of individual $\mathrm{T}_{\mathrm{NEAR}}$ and $\mathrm{T}_{\mathrm{FAR}}$ delays for the $\mathrm{fMRI}$ session" section), we wanted to check that differences in the experimental set up between the fMRI and the behavioral sessions did not affect the perceived distance of the sound sources. We ran a sound localization experiment on a new group of participants. During the experiment, they were blindfolded. The experiment consisted of two blocks randomly administered. In one block (Block B), participants sat down with their right arm resting palm down on a table and received auditory stimulation by loudspeakers (as in the behavioral audio-tactile interaction task); in a second block (Block F), they laid down in a mock scanner with their right arm resting at their side and received auditory stimulation by headphones (as in the fMRI audio-tactile interaction task). In both blocks, they received a tactile stimulation on the right index finger at one of the five different temporal delays from the sound onset (T1-T5). Each participant was presented with a random combination of 12 target stimuli for each temporal delay for the looming and flat sounds in each block. At the end of each trial, participants were required to verbally indicate the perceived position of the sound in space when they had felt the tactile stimulus on a scale from 1 (very close) to 100 (very far). They were explicitly encouraged to use the entire range between 1 and 100 (for a similar procedure, see Canzoneri et al., 2012; Ferri et al., 2015).

\section{fMRI session}

\section{Auditory task}

Participants were blindfolded and asked to keep their eyes closed. They listened passively to looming and flat sounds, which corresponded to the auditory component of the multisensory stimuli presented during the PPS task. The auditory stimuli occurred randomly every $4.65,6.2$, or $7.75 \mathrm{~s}$ (corresponding to 3, 4, and 5 magnetic resonance (MR) time points, respectively). Each participant underwent 2 runs of 6 min each for a total of 30 auditory stimuli for each condition (looming and flat) equally distributed across the two runs (Fig. 2, top row).

\section{fMRI audio-tactile interaction task (PPS)}

Participants were blindfolded and asked to keep their eyes closed. During each trial, either a looming or a flat sound was presented. Along with the auditory stimulation, participants were always presented with a tactile stimulus. The tactile stimulus could be delivered on either their right (experimental trials) or their left (catch trials) index finger and at two different temporal delays $\left(T_{\mathrm{NEAR}}\right.$ and $T_{\mathrm{FAR}}$ ) from the sound onset. $T_{\mathrm{NEAR}}$ and $T_{\text {FAR }}$ were defined individually for each participant as described above. However, between-subjects differences for both $T_{\text {NEAR }}$ and $T_{\mathrm{FAR}}$ were $<1$ MR time point. Four different experimental conditions resulted from the interaction between the two types of sounds (looming, flat) and tactile temporal delays $\left(T_{\mathrm{NEAR}}, T_{\mathrm{FAR}}\right): L T_{\mathrm{NEAR}}=$ looming sound $/ T_{\mathrm{NEAR}}$; $L T_{\mathrm{FAR}}=$ looming sound $/ T_{\mathrm{FAR}} ; F T_{\mathrm{NEAR}}=$ flat sound $/ T_{\mathrm{NEAR}} ; F T_{\mathrm{FAR}}=$ flat sound $/ T_{\text {FAR }}$ (Fig. 1, bottom row). Each trial was followed by a variable intertrial time $(4.65,6.2$, or $7.75 \mathrm{~s}$, corresponding to 3,4 , and $5 \mathrm{MR}$ time points, respectively).

Participants were asked to respond as fast as possible to the tactile target only when it was delivered on their left index finger (catch trials), trying to ignore the auditory stimulus. To give a response, they had to press a button on a response box (MRI-compatible response pad Lumina LU400 and LSC-400 controller; Cedrus) with their left middle finger. These trials had the purpose of keeping participants awake and alert. To rule out the possibility that button pressing affected BOLD responses during experimental trials, catch trials were always followed by an additional time interval ( $1.55 \mathrm{~ms}$, corresponding to $1 \mathrm{MR}$ time point).

Each participant was presented with a random combination of 30 experimental trials for each condition $\left(L T_{\mathrm{NEAR}}, L T_{\mathrm{FAR}}, F T_{\mathrm{NEAR}}, F T_{\mathrm{FAR}}\right)$ randomly intermingled with 20 catch trials for the same conditions. The 4 conditions and the 2 types of trial (experimental, catch) were equally distributed across 5 runs of 6 min each. 


\section{Image acquisition}

A 3 T Philips Achieva scanner (Institute of Advanced Biomedical Technologies, Chieti, Italy) was used to acquire MRI data using a whole-body radiofrequency coil for signal excitation and an eight-channel phasedarray head coil for signal reception. BOLD contrast images over the entire brain were acquired with a gradient-echo echoplanar sequence [repetition time (TR), $1550 \mathrm{~ms}$; echo time (TE), $30 \mathrm{~ms} ; 29$ axial slices with a 0.5 $\mathrm{mm}$ gap; slice thickness, $3 \mathrm{~mm}$; in-plane resolution, $3 \times 3 \mathrm{~mm}$ ].

Anatomical images were acquired via a $3 \mathrm{D}$ fast-field echo T1weighted sequence $(1 \mathrm{~mm}$ isotropic voxel size, TR/TE $=8.1 / 3.7 \mathrm{~ms}$, flip angle $=8^{\circ}$ ).

\section{Data analysis}

$f M R I$ data preprocessing

Preprocessing steps of functional data were implemented in AFNI (Cox, 1996) (http://afni.nimh.nih.gov/afni). Functional images were realigned within and across runs to correct for head motion using six-parameter rigid-body realignment. Slice-timing correction was applied to remove differences in acquisition times between slices. The high-resolution anatomical image and the functional images were coregistered and stereotactically normalized to Talairach space (Talairach and Tournoux, 1988). Functional images were spatially smoothed with a 3D Gaussian filter of 6 mm full-width-at-half-maximum.

\section{Definition of ROIs}

To avoid the circularity that can arise from the use of the same dataset for selection and selective analysis (Kriegeskorte et al., 2009), we defined ROIs based on their sensitivity to the looming sounds, as revealed by the auditory task, and their involvement in PPS representation, as known from previous fMRI studies (Makin et al., 2007; Brozzoli et al., 2011, 2013). Therefore, the selection was made independently from the audiotactile interaction task. Indeed, we wanted to avoid using trial-averaged BOLD responses to near and far stimuli to both select regions coding PPS and look for neural predictors of individual PPS boundaries. This choice was further prompted by the fact that trial-averaged BOLD responses and their ITV can be related (He, 2013). The reason for combining the two criteria was that most of the neuroimaing studies on PPS focused on visuo-tactile interaction (Makin et al., 2007; Brozzoli et al., 2011, 2013; Serino et al., 2011, but see also Bremmer et al., 2001). Therefore, there is no previous evidence showing that brain regions involved in PPS representation are also sensitive to approaching auditory stimuli.

Following the above described preprocessing procedures, the data from the auditory task were submitted to a standard GLM analysis to obtain a map of the estimated coefficients for the two auditory conditions (looming, flat). Group analyses were then performed to produce the contrast [looming sound] - [flat sound]. This contrast allowed identifying voxels responding higher to looming than flat sounds. The resulting group-level $t$-map was thresholded at $p<0.005$. To correct for multiple comparisons, we used Monte Carlo simulation as implemented in the AFNI program AlphaSim, yielding a familywise error rate (FWER) at $p<$ 0.05 (Xiong et al., 1995).

We then defined PPS ROIs based on the overlap between the grouplevel $t$-map and regions previously reported to play a crucial role in PPS representation, such as premotor and parietal regions (Makin et al., 2007; Brozzoli et al., 2011, 2013). We designed the PPS ROIs around the coordinates taken from the literature about PPS (Makin et al., 2007; Brozzoli et al., 2011, 2013), which better overlapped with the looming-sensitive brain voxels identified by the group-level $t$-map.

In addition, we defined auditory ROIs centered on the local maxima of the group-level $t$-map to control for the specificity of spatial modulation of brain responses in PPS ROIs. ROIs were $6 \mathrm{~mm}$ radius spheres.

A whole-brain, voxelwise approach was then used to confirm that the ROI selection criteria that we adopted considered all the brain regions coding PPS that were involved in the audio-tactile interaction task. For each participant and condition of the experimental trials ( $L T_{\mathrm{NEAR}}$, $\left.L T_{\mathrm{FAR}}, F T_{\mathrm{NEAR}}, F T_{\mathrm{FAR}}\right)$, the whole-brain $\beta$ coefficient maps were used to perform the following contrast: $\left[L T_{\mathrm{NEAR}}-F T_{\mathrm{NEAR}}\right]-\left[L T_{\mathrm{FAR}}-\right.$ $\left.F T_{\mathrm{FAR}}\right]$. The resulting group-level map was thresholded at $p<0.005$. To correct for multiple comparisons, we used Monte Carlo simulation as implemented in the AFNI program AlphaSim, yielding an FWER at $p<$ 0.05 (Xiong et al., 1995).

\section{Time course analyses and statistical tests}

Following the above preprocessing procedures, BOLD data from the PPS task runs for each participant were subsequently submitted to regression analyses using a finite impulse response (FIR) model, as implemented in AFNI via the TENTzero basis function. Trials were modeled by a set of 7 functions covering 7 consecutive MR time points, each $1.55 \mathrm{~s}$, aligned with the onset of the stimulus. Seven MR time points were enough to cover the peak of BOLD responses, with no overlap across trials (Fox et al., 2006; Fox et al., 2007; He, 2013). Each trial type ( $L T_{\mathrm{NEAR}}, L T_{\mathrm{FAR}}$, $F T_{\mathrm{NEAR}}$, and $F T_{\mathrm{FAR}}$ conditions for experimental and the catch trials), as well as false alarms (for experimental trials) and missed responses (for catch trials), were modeled separately. This procedure estimated hemodynamic response patterns ( $\beta$ coefficient maps) for each of the seven MR time points after stimulus onset and for each condition. Motion parameters obtained during head motion correction were used as additional nuisance regressors.

For each participant and ROI, regional BOLD time course for each condition ( $\left.L T_{\mathrm{NEAR}}, L T_{\mathrm{FAR}}, F T_{\mathrm{NEAR}}, F T_{\mathrm{FAR}}\right)$, only for experimental trials, were estimated over seven MR time points by averaging across all the voxels in a given ROI. Finally, for each participant, ROI, and condition, we used the mean $\beta$ coefficient of the peak defined by the group-averaged time course, and its two adjacent time points to quantify the task-evoked BOLD signal changes. This averaging procedure is usually applied to denoise fMRI data. Groupwise statistics were performed on these threepoint-averaged peak values.

For each ROI, we performed the contrast $\left[L T_{\mathrm{NEAR}}-F T_{\mathrm{NEAR}}\right]-$ $\left[L T_{\mathrm{FAR}}-F T_{\mathrm{FAR}}\right]$ to assess its involvement in PPS representation. To control for possible confounding effects due to individual differences in the location of PPS boundary, we used individual CP values as a covariate. A significance threshold of $p<0.05$, corrected for the number of tested ROIs, was adopted for this analysis. Only participants with a statistically acceptable estimation of the $\mathrm{CP}$ were included in this analysis (acceptance of CP: $p<0.05$; Table 1).

\section{ITV analysis}

Following the above preprocessing procedures, model-free BOLD data for each participant, ROI, and condition $\left(L T_{\mathrm{NEAR}}, L T_{\mathrm{FAR}}, F T_{\mathrm{NEAR}}, F T_{\mathrm{FAR}}\right.$ and also looming sound) were epoched at seven MR time points after stimulus onset by averaging across all voxels in the ROI. We chose the epoch length according to the FIR analysis (see above). SD across all trials was computed at each MR time point for each participant, ROI, and condition. The SD time course for each participant, ROI, and condition was normalized to the first frame (He, 2013) because we were interested in quantifying the stimulus-induced change of variability of brain activity compared with its level at the stimulus onset. Finally, three-pointaveraged SD values for each participant were computed around the corresponding group peaks as defined in the preceding analyses (see "Time courses analyses and statistical tests" section) for each ROI and condition. Groupwise statistics were performed on these three-point-averaged ITV values. Only participants with a statistically acceptable estimation of the CP were included in these analyses (acceptance of CP: $p<0.05$ ).

We preliminarily checked that the timing of stimulus presentation during the PPS task allowed stimuli to eventually induce ITV reduction. For this, we analyzed ITV profiles in different brain regions, including the control auditory region that we here report as a proof. Two-tailed onesample $t$ tests on 3-point-averaged ITV values showed significant ITV reduction for different conditions $\left(L T_{\mathrm{FAR}}: t_{35}=-2.126, p=0.041\right.$; $\left.L T_{\mathrm{NEAR}}: t_{35}=-2.634, p=0.012 ; F T_{\mathrm{FAR}}: t_{35}=-3.953, p<0.001\right)$.

\section{Contribution of ITV and averaged BOLD responses to individual} PPS boundary $(C P)$

We performed Pearson's correlation analyses to investigate the predictive power of either averaged BOLD responses or ITV for individual PPS boundary. Either the three-point-averaged peak values or the corresponding three-point-averaged ITV values for different experimental conditions were correlated with individual values of CP. Then, we separated our participants into two groups according to their $\mathrm{CP}$ by a median 
Table 1. CP of the individual subjects and goodness of fit of the sigmoidal distribution of RTs

\begin{tabular}{|c|c|c|c|c|c|c|}
\hline \multirow[b]{2}{*}{ Subject } & \multirow[b]{2}{*}{$C P$} & \multicolumn{2}{|c|}{ Acceptance of CP estimates } & \multicolumn{3}{|c|}{ Goodness of fit } \\
\hline & & $t$ value & $p$ value & $R^{2}$ & MSE & NRMSE \\
\hline 1 & 1884 & 64.7 & 4.1E-06 & 0.9975 & 0.0003 & 0.115 \\
\hline 2 & 2000 & 32.4 & $3.2 \mathrm{E}-05$ & 0.9929 & 0.0001 & 0.224 \\
\hline 3 & 1760 & 26.8 & 5.7E-05 & 0.9878 & 0.0007 & 0.502 \\
\hline 4 & 1756 & 22.4 & $9.5 \mathrm{E}-05$ & 0.9822 & 0.0002 & 0.129 \\
\hline 5 & 1386 & 19.6 & $1.4 \mathrm{E}-04$ & 0.9948 & 0.0001 & 0.171 \\
\hline 6 & 972 & 16.5 & $2.4 \mathrm{E}-04$ & 0.9876 & 0.0001 & 0.212 \\
\hline 7 & 2241 & 14.1 & $3.9 \mathrm{E}-04$ & 0.9616 & 0.0001 & 0.300 \\
\hline 8 & 1120 & 12.3 & 5.0E-04 & 0.9765 & 0.0034 & 0.285 \\
\hline 9 & 2007 & 9.7 & $1.5 \mathrm{E}-03$ & 0.8836 & 0.0112 & 0.106 \\
\hline 10 & 1115 & 9.4 & $1.0 \mathrm{E}-03$ & 0.9616 & 0.0024 & 0.386 \\
\hline 11 & 2061 & 9.2 & $1.5 \mathrm{E}-03$ & 0.8516 & 0.0121 & 0.245 \\
\hline 12 & 1966 & 9.0 & $1.5 \mathrm{E}-03$ & 0.8724 & 0.0033 & 0.250 \\
\hline 13 & 1458 & 8.9 & $1.5 \mathrm{E}-03$ & 0.9362 & 0.0033 & 0.072 \\
\hline 14 & 1496 & 7.8 & 2.0E-03 & 0.9149 & 0.0028 & 0.099 \\
\hline 15 & 1182 & 7.3 & $2.5 \mathrm{E}-03$ & 0.9279 & 0.0059 & 0.296 \\
\hline 16 & 1859 & 7.2 & $2.5 \mathrm{E}-03$ & 0.8457 & 0.0082 & 0.296 \\
\hline 17 & 1160 & 6.8 & 3.0E-03 & 0.9077 & 0.0048 & 0.622 \\
\hline 18 & 1228 & 6.5 & $3.5 \mathrm{E}-03$ & 0.9037 & 0.0089 & 0.337 \\
\hline 19 & 1366 & 6.2 & 4.0E-03 & 0.8872 & 0.0047 & 0.242 \\
\hline 20 & 1545 & 6.2 & 4.0E-03 & 0.8036 & 0.0091 & 0.200 \\
\hline 21 & 1509 & 6.1 & $4.5 \mathrm{E}-03$ & 0.8231 & 0.0058 & 0.047 \\
\hline 22 & 774 & 6.0 & $4.5 \mathrm{E}-03$ & 0.7401 & 0.0035 & 0.385 \\
\hline 23 & 785 & 5.5 & $6.0 \mathrm{E}-03$ & 0.7506 & 0.0031 & 0.703 \\
\hline 24 & 1538 & 5.3 & 5.0E-03 & 0.7943 & 0.0165 & 0.436 \\
\hline 25 & 1196 & 5.0 & 7.5E-03 & 0.8639 & 0.0083 & 0.129 \\
\hline 26 & 1749 & 4.9 & 1.0E-02 & 0.5976 & 0.0149 & 0.261 \\
\hline 27 & 1772 & 4.8 & $1.0 \mathrm{E}-02$ & 0.7257 & 0.0068 & 0.279 \\
\hline 28 & 870 & 2.8 & $3.3 \mathrm{E}-02$ & 0.7543 & 0.0023 & 0.404 \\
\hline
\end{tabular}

$(\mathrm{C}$, Central point; MSE, mean squared error; NRMSE, normalized root mean squared error [RMSE/(ymax $-y \min )]$.

split. One group, the $\mathrm{CP} w$ group, was characterized by low $\mathrm{CP}$ values corresponding to a distant-from-the body location of PPS boundary; that is, a wide PPS. The other group, the $\mathrm{CP} n$ group, was characterized by high $\mathrm{CP}$ values indicating that their PPS boundary was located near to their body; that is, narrow PPS. One-sample $t$ tests against zero assessed significant modulations of ITV in each group and condition.

We performed two mixed ANOVAs with group $(\mathrm{CP} w, \mathrm{CP} n)$ as the between-subjects factor and space $\left(L T_{\mathrm{NEAR}}, L T_{\mathrm{FAR}}\right)$ as the withinsubjects factor on either ITV or averaged BOLD responses. Simple effect analyses tested for between- and within-group differences. In all of the above-described analyses, we used Bonferroni's correction for multiple comparisons. Only participants with a statistically acceptable estimation of the CP were included in these analyses (acceptance of CP: $p<0.05$ ).

\section{Results}

\section{Behavioral studies}

Behavioral audio-tactile interaction task: evidence of individual differences of the PPS boundary

Participants were asked to respond as fast as possible to a tactile target delivered on their right index finger, along with a taskirrelevant sound (Fig. 1a). RTs were log-transformed because of the non-normal distribution of their values. Mean logtransformed RTs to tactile targets were calculated separately for each experimental condition. On average, a total of $97.5 \pm 3.5 \%$ $( \pm \mathrm{SD})$ from each participant were included in data analyses. Rate of omissions were $2.1 \%$ and $2.8 \%$ for flat and looming trials, respectively. Responses longer than 2 SDs from the individual mean were treated as outliers and not considered further $(2.1 \%$ for flat and $2.0 \%$ for looming trials). Specific modulations of tactile RTs induced by the perceived position of approaching sounds compared with flat sounds were assessed by ANOVA. The critical two-way interaction was significant $\left(F_{(4,140)}=33.06 ; p<\right.$ 0.001 ). This entailed specific modulation of RTs due to the per- ceived position of approaching compared with flat sounds in space. Tactile RTs were significantly slower in case of looming sounds compared with the flat sounds at T1 $(5.91 \pm 0.14$ vs $5.84 \pm 0.15 \mathrm{~ms}$, respectively), whereas they were faster at $\mathrm{T} 4$ ( $5.75 \pm 0.14$ vs $5.82 \pm 0.15 \mathrm{~ms}$, respectively) and T5 (5.71 \pm 0.16 $\mathrm{ms}$ vs $5.81 \pm 0.13 \mathrm{~ms}$, respectively) ( $p<0.001$ in all cases, Bonferroni's correction). Moreover, in cases of looming stimuli, RTs were faster when sounds were perceived at T4 and T5 (5.75 \pm 0.14 and $5.71 \pm 0.16 \mathrm{~ms}$, respectively) than at T1, T2, or T3 $(5.91 \pm 0.14,5.87 \pm 0.13$, and $5.80 \pm 0.14 \mathrm{~ms}$, respectively; $p<$ 0.001 in all cases, Bonferroni's correction). The same modulation did not apply to log-transformed RTs for the flat sounds.

Next, we estimated the individual differences in the location of PPS boundary. First, for each participant, the mean RTs to the tactile targets delivered along with looming sounds were fitted to a sigmoidal function (Fig. 1a). Consistent with previous literature (Canzoneri et al., 2012; Teneggi et al., 2013; Ferri et al., 2015), this model provided a better description of the relationship between tactile RTs and timing at which the tactile stimuli were delivered compared with a linear model. Indeed, the RMSE was lower for the sigmoid $(0.066 \pm 0.037 \mathrm{~ms})$ than the linear model $\left(0.080 \pm 0.036 \mathrm{~ms} ; t_{35}=-3.98, p<0.001\right)$. Next, to estimate the individual boundaries of PPS representation, we computed the CP of the sigmoid curve for each participant. The $\mathrm{CP}$ is defined as the value of the abscissa at the CP of the sigmoid and indicates the individual location of the PPS boundary (Canzoneri et al., 2012; Teneggi et al., 2013; Ferri et al., 2015). $t$ test analysis on individual CP estimates showed statistical relevance $(p<0.05)$ of the parameter of interest for 28 participants (see Table 1 for $t$-statistics and goodness-of-fit indexes). The average $\mathrm{CP}$ for these participants was $1491 \pm 411 \mathrm{~ms}$ ranging from 774 to $2241 \mathrm{~ms}$ (Fig. 1a). Therefore, the computation of the individual $\mathrm{CP}$ revealed high differences across participants (Fig. 1b, Table 1).

The problem was how to translate the $\mathrm{CP}$ in milliseconds into its spatial location in centimeters. It is known that high CPs in milliseconds mirror a narrow PPS, whereas low CPs in milliseconds mirror a wide PPS (Canzoneri et al., 2012; Teneggi et al., 2013; Ferri et al., 2015). To allow a more precise conversion of the temporal dimension of the paradigm to the location of sounds and CPs in space, we first identified the exponential function, which better describes our looming sounds. To this aim, we used the formula $a * \exp (b * x)$, then we computed the intensity of the sound at each point of the exponential function. Based on these parameters and assuming the speed of sound as constant, we calculated that our five tactile stimuli were delivered when the approaching sound was at $97.7 \mathrm{~cm}$ (T1), $95.3 \mathrm{~cm}(\mathrm{~T} 2)$, $88.6 \mathrm{~cm}$ (T3, very close to the average CP), $70.5 \mathrm{~cm}$ (T4), and 41.7 $\mathrm{cm}$ (T5) from the participant's hand.

\section{Reproducibility of the CP across sessions}

This behavioral study was performed to test for the reproducibility of individual CP values across sessions. Sixteen of 20 participants had a good sigmoidal fit (acceptance of CP: $p<0.05$ ) in both sessions. Their results showed that reproducibility of $\mathrm{CP}$ measures over time is acceptable, even when experimental sessions are conducted a week or more apart. Indeed, when we tested for the reproducibility of group mean values, we found no difference in the mean distribution of CPs between sessions (Session 1 $\mathrm{CP}=1598$; Session $2 \mathrm{CP}=1561 ; t_{15}=0.47 ; p=0.64$ ). Moreover, when we tested for the reproducibility of individual values, we found a significant correlation between the CPs from the two sessions $[r=0.73 ; p<0.001$; bootstrap confidence interval $(\mathrm{CI})$ : $0.44-0.90]$. 
Table 2. Perceived position of sounds in space

\begin{tabular}{llllll}
\hline & \multicolumn{3}{l}{ Block B } & & Block F \\
\cline { 2 - 3 } \cline { 5 - 6 } Distance & Flat \% (SE) & Looming \% (SE) & & Flat \% (SE) & Looming \% (SE) \\
\hline T1 & $39.0(5.9)$ & $81.9(4.9)$ & & $32.0(6.7)$ & $74.7(5.3)$ \\
T2 & $37.8(4.1)$ & $69.3(3.6)$ & & $30.4(5.1)$ & $63.4(3.8)$ \\
T3 & $36.7(3.3)$ & $49.1(3.5)$ & & $28.4(3.4)$ & $40.8(3.0)$ \\
T4 & $34.3(3.8)$ & $25.3(3.5)$ & & $25.9(3.8)$ & $21.4(3.4)$ \\
T5 & $32.8(4.7)$ & $14.9(4.1)$ & & $25.2(5.0)$ & $12.5(4.3)$ \\
\hline
\end{tabular}

For Block B, participants sat down with their right arm resting palm down on a table and received auditory stimulation by loudspeakers (as in the behavioral audio-tactile interaction task). For Block F, participants laid down in a mock scanner with their right arm resting at their side and received auditory stimulation by headphones (as in the fMRI audio-tactile interaction task). $1 \%$ means very close; $100 \%$, very far.

\section{Reproducibility of the CP across tasks}

This behavioral study was performed to test for the reproducibility of individual CP values across tasks. Specifically, this study aimed at ruling out the possibility that the adaptation of the behavioral PPS task for the fMRI session may affect participants' PPS boundaries. Two participants were discarded because of bad fit of the sigmoid function (acceptance of CP: $p<0.05$ ). The following analyses were run on the remaining 18 participants. We first tested for differences in the location of the CPs. To this aim, a paired-sample $t$ test was run between CPs measured during the first and the second study. This analysis did not reveal any difference in the mean distribution of CPs between experiments (Experiment $1 \mathrm{CP}=1467 \mathrm{~ms}$; Experiment $2 \mathrm{CP}=1578 \mathrm{~ms} ; t_{17}=$ $1.17 ; p=0.26)$. As a second step, we looked for correlations between the CPs from the two experiments. Results revealed a positive correlation $(r=0.55$; $p=0.018$; bootstrap CI: $0.25-$ 0.88 ); participants with a wide $\mathrm{CP}$ in the first experiment also showed a wide CP in the second experiment.

\section{Sound localization task}

The aim of this study was to demonstrate that differences in the experimental setup between the fMRI session and the behavioral session, used to define participants' $\mathrm{CP}$, did not affect the perceived distance of the sound sources. Regarding looming sounds, the pattern of responses was the same for the two experimental blocks (Block B and Block F) because participants progressively perceived them to be closer to their body from T1 to T5 in both cases. In contrast, for flat sounds, there was no modulation of the responses across temporal delays in either block (Table 2). Results from a 2 (block) $\times 2$ (sound) $\times 5$ (temporal delays) ANOVA confirmed these observations. Indeed, the factor block did not significantly interact with any other factor: block $\times$ sound $\left(F_{(1,19)}=1.856, p=0.189\right)$; block $\times$ temporal delays $\left(F_{(4,76)}=0.52, p=0.721\right)$; block $\times$ sound $\times$ temporal delays $\left(F_{(4,76)}=0.826, p=0.512\right)$. Only the interaction sound $\times$ temporal delays was significant $\left(F_{(4,76)}=75.039, p<0.001\right)$. These results clearly suggest that differences between the fMRI and the behavioral sessions concerning participants' posture and auditory stimulus delivery did not affect the perceived distance of the sound sources.

\section{fMRI study}

Two participants (from the original $n=38$ ) were eliminated due to excessive movement during scanning. Data from the residual 36 volunteers were included in the whole-brain fMRI analyses not using CP values. Only the subgroup of participants showing statistically acceptable CP estimates $(n=28)$ were included in the ROI-based fMRI analyses assessing the relationship between individual CP and either ITV or trial-averaged BOLD responses.

First, to further legitimize the choice of the temporal delays in the $T_{\mathrm{NEAR}}$ and $T_{\mathrm{FAR}}$ conditions for each participant, we analyzed log-transformed RTs in response to catch trials recorded during the fMRI session. As expected, we found a systematic difference between log-transformed RTs to $T_{\mathrm{NEAR}}$ and $T_{\mathrm{FAR}}(5.96 \pm 0.28 \mathrm{vs}$ $\left.6.14 \pm 0.24 \mathrm{~ms} ; t_{35}=6.663, p<0.001\right)$ with no participant showing faster RTs to the far condition compared with the near.

\section{Brain regions involved in audio-tactile PPS representation}

Because the PPS representation rested on participants' perception of approaching stimuli, we independently defined our ROIs as regions showing both significant activation during looming sound (looming sensitivity) in our data and the involvement in PPS representation from previously reported foci.

To look for looming-sensitive brain regions, we applied the following contrast in the unimodal runs: [looming sound] - [flat sound]. This showed activation of voxels in the left PM and inferior frontal gyrus, as well as in the bilateral superior temporal sulcus/middle temporal gyrus (Fig. $3 a$ ). These results are consistent with those obtained by Seifritz et al. (2002), which compared fMRI signals in response to looming and receding pure tones.

Then, we defined PPS ROIs based on the overlap between contrast results and regions previously reported to play a crucial role in PPS representation (Makin et al., 2007; Brozzoli et al., 2011, 2013). Accordingly, we defined a $6 \mathrm{~mm}$ radius spherical ROI in the PM around the Talairach coordinates $[-41 ;-1 ; 25]$ taken from Brozzoli et al. (2013) because they showed the optimal overlap with looming-sensitive premotor voxels.

To examine the specificity of audio-tactile interaction in brain regions involved in PPS representation, we defined a 6-mmradius sphere in the left STG/MTG as the control region. This auditory ROI (Fig. $3 a$ ) was centered on the peak voxel (left hemisphere: $-37 ;-31 ; 14)$. We then used these two ROIs, namely the premotor and the auditory cortex, in all subsequent analyses assessing the predictive role of ITV compared with trial-averaged amplitude of BOLD responses for individual PPS boundaries.

First, however, we performed a whole-brain analysis to verify that our independent selection of the ROIs did not omit any potential PPS-coding region. Based on the behavioral evidence that looming sounds specifically facilitate audio-tactile interactions in the perceived near space (Fig. 1a), we hypothesized that brain regions involved in PPS representation would show varying responses to audio-tactile interaction depending on whether the sound is perceived as being in the near or far space. For this purpose, we applied the following contrast to BOLD data: $\left[L T_{\text {NEAR }}-F T_{\text {NEAR }}\right]-\left[L T_{\text {FAR }}-F T_{\text {FAR }}\right]$. The wholebrain analysis identified a significant cluster of 161 voxels in the left precentral gyrus (Talairach coordinates: $-49-10$ 20; Fig. $3 d$ ) partially overlapping with the left PM ROI. This result further supported the role of this region in the representation of audiotactile PPS (see also the next paragraph). Another significant cluster (244 voxels) was centered in the right medial frontal gyrus (Talairach coordinates: 16753 ). These results ruled out the possibility that a restrictive selection of the ROIs prevented us from finding a role for other potential PPS-coding regions (the posterior parietal cortex) in the prediction of individual audio-tactile PPS boundary.

\section{Audio-tactile PPS in the premotor ROI}

To demonstrate that the independently selected PM ROI was involved in PPS representation, we performed the contrast $\left[L T_{\mathrm{NEAR}}-F T_{\mathrm{NEAR}}\right]-\left[L T_{\mathrm{FAR}}-F T_{\mathrm{FAR}}\right]$ in this region, as well as in the control auditory ROI. To account for possible confounding effects due to behavioral differences between participants (Fig. 1), we included the individual $\mathrm{CP}$ values as a control variable. In the 


\section{Looming sound vs. Flat sound}

\section{a}
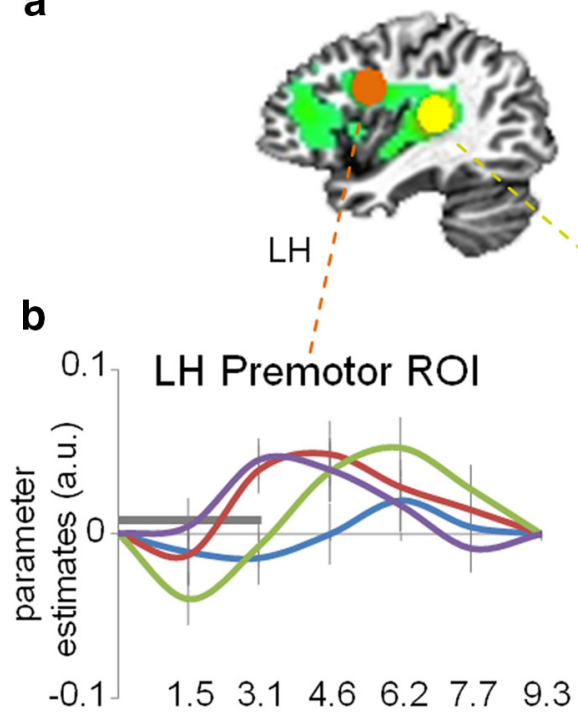

$\longrightarrow \mathrm{LT}_{\mathrm{NEAR}}=\mathrm{LT}_{\mathrm{FAR}}$
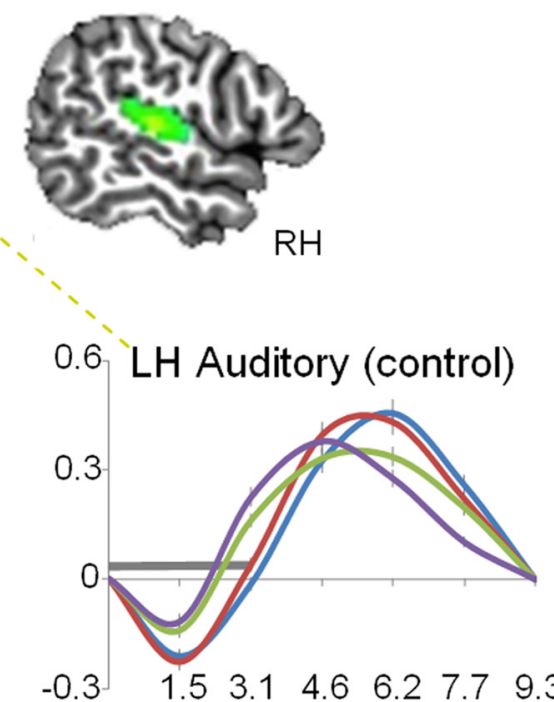

\section{Near vs. Far}
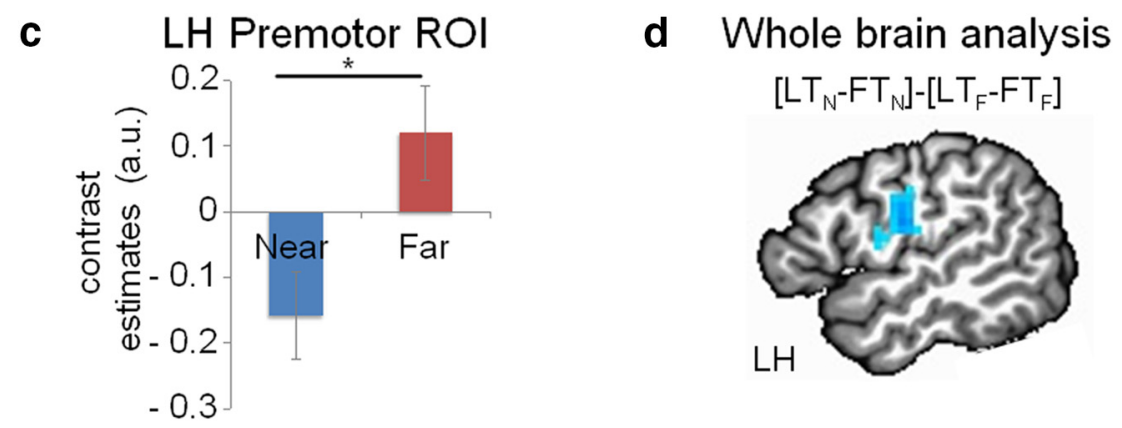

Figure 3. Selection of the ROls: looming sensitive and involved in PPS representation. $\boldsymbol{a}$, First, we identified looming-sensitive brain voxels (green) by applying the contrast [looming sound] - [flat sound] to the whole brain. Second, we defined PPS ROIs based on the overlap between contrast results and brain regions previously reported to play a crucial role in PPS representation, such as the PM (Brozzoli et al., 2013; orange sphere). The left auditory ROI (yellow sphere), centered on the local maximum, was taken as a control region. $\boldsymbol{b}$, Group-averaged BOLD signal time courses for each condition of the PPS task $\left(L T_{\mathrm{NEAR}} L T_{\mathrm{FAR}}, F T_{\mathrm{NEAR}}\right.$ $\left.F T_{F A R}\right)$. Vertical bars indicate SD; gray horizontal bars indicate the stimulus duration. $C$, ROI-based analysis to test the role of PM ROI in coding PPS. Plots of the contrast estimates assessing the impact of looming sounds on audio-tactile interaction in the near $\left(\left[L T_{\text {NEAR }}-F T_{\text {NEAR }}\right]\right)$ and far $\left(\left[L T_{\text {FAR }}-F T_{\text {FAR }}\right]\right)$ conditions. Vertical bars indicate SD. After partialling out the individual differences in $C P$, the near and far conditions differed significantly $\left({ }^{* * *} p \leq 0.001\right)$. $d$, Whole-brain analysis confirming the role of PM in coding PPS. Shown is the whole-brain statistical map from the contrast $\left[L T_{\mathrm{NEAR}}-F T_{\mathrm{NEAR}}\right]-\left[L T_{\mathrm{FAR}}-F T_{\mathrm{FAR}}\right](p<0.05$ corrected).

left PM, we clearly observed a spatial modulation of BOLD responses as the controlled difference between contrast estimations for near $\left(\left[L T_{\mathrm{NEAR}}-F T_{\mathrm{NEAR}}\right]\right)$ and far $\left(\left[L T_{\mathrm{FAR}}-F T_{\mathrm{FAR}}\right]\right)$ conditions was significant $\left(F_{26}=6.916 ; p=0.014\right.$, Bonferroni's correction for the number of regions evaluated; Fig. $3 c$ ). This result indicates that, after partialling out the individual differences in the CP, premotor responses to audio-tactile interactions significantly differ according to the perceived position of the looming sound. In other words, the PM discriminates between environmental stimuli occurring at near and far distances. This evidence is consistent with Brozzoli et al.'s (2013) findings about the role of this premotor region in PPS representation. In contrast, in the auditory ROI, the spatial modulation of responses to audio-tactile interactions did not reach statistical significance $\left(F_{26}=0.604 ; p=0.444\right)$. (s)

As can be observed in Figure $3 c$, the $\mathrm{BOLD}$ response in the $\mathrm{PM}$ was higher in the far condition compared with the near. How should we interpret this finding? Neuroimaging studies investigating multisensory interaction in humans show that BOLD responses to multisensory stimuli can change as a function of both task difficulty and stimulus quality (James et al., 2012; Noppeney, 2012). Specifically, as the difficulty of the task increases (Kim et al., 2012) or the effectiveness of the constituent unisensory stimuli lowers (Werner and Noppeney, 2010), the multisensory enhancement increases. This in turn positively affects the BOLD response. Our results showing higher premotor activity evoked by the $L T_{\mathrm{FAR}}$ compared with $L T_{\text {NEAR }}$ condition are in agreement with these previous findings (Fig. $3 b, c$ ). Indeed, the $L T_{\mathrm{FAR}}$ condition is associated with both increased task difficulty, as suggested by slower RTs during catch trials, and modest effectiveness of the auditory stimulus due to the low intensity of the sound when the tactile stimulus arrives.

\section{$B O L D$ response variability (ITV) and individual behavioral differences}

Recent studies have revealed that the variability of BOLD responses is not simply "noise" (Garrett et al., 2013b; Zilles and Amunts, 2013). Measures of BOLD response variability seem to provide promising tools to predict behavior (Fox et al., 2007; He, 2013), as well as individual characteristics such as chronological age (Garrett et al., 2010). Based on these suggestions, we tested whether ITV in the left PM might account for individual differences in PPS extension.

To this aim, we performed Pearson's correlation analyses between BOLD response variability (ITV) and individual locations of PPS boundary (CP). Only ITV in the far space condition, $L T_{\mathrm{FAR}}$, specifically predicted individual CP values $(r=0.548, p=0.003$; bootstrap CI: $0.367-0.719)$. In contrast, the correlation of ITV in the near space condition, $L T_{\mathrm{NEAR}}$, with $\mathrm{CP}$ was not significant $(r=$ $-0.149, p=0.45$; Fig. 4$)$. ITV during audio-tactile interactions involving flat sounds $\left(F T_{\mathrm{FAR}}\right.$ and $\left.F T_{\mathrm{NEAR}}\right)$ also did not correlate with CP $\left(F T_{\mathrm{FAR}}: r=-0.035, p=0.858\right.$ and $F T_{\mathrm{NEAR}}$ : $r=-0.035, p=0.858)$ as well. The auditory ROI provided a control for the anatomical specificity of our result. ITV during $L T_{\mathrm{FAR}}$ did not show any predictive power for the CP in this region $(r=0.172, p=0.381)$. Finally, there was no significant correlation between ITV induced in left PM by looming sound alone and the CP $(r=-0.094, p=0.634)$, suggesting that the interaction between looming sounds and tactile stimuli is necessary for inducing ITV modulation predictive for the CP. 


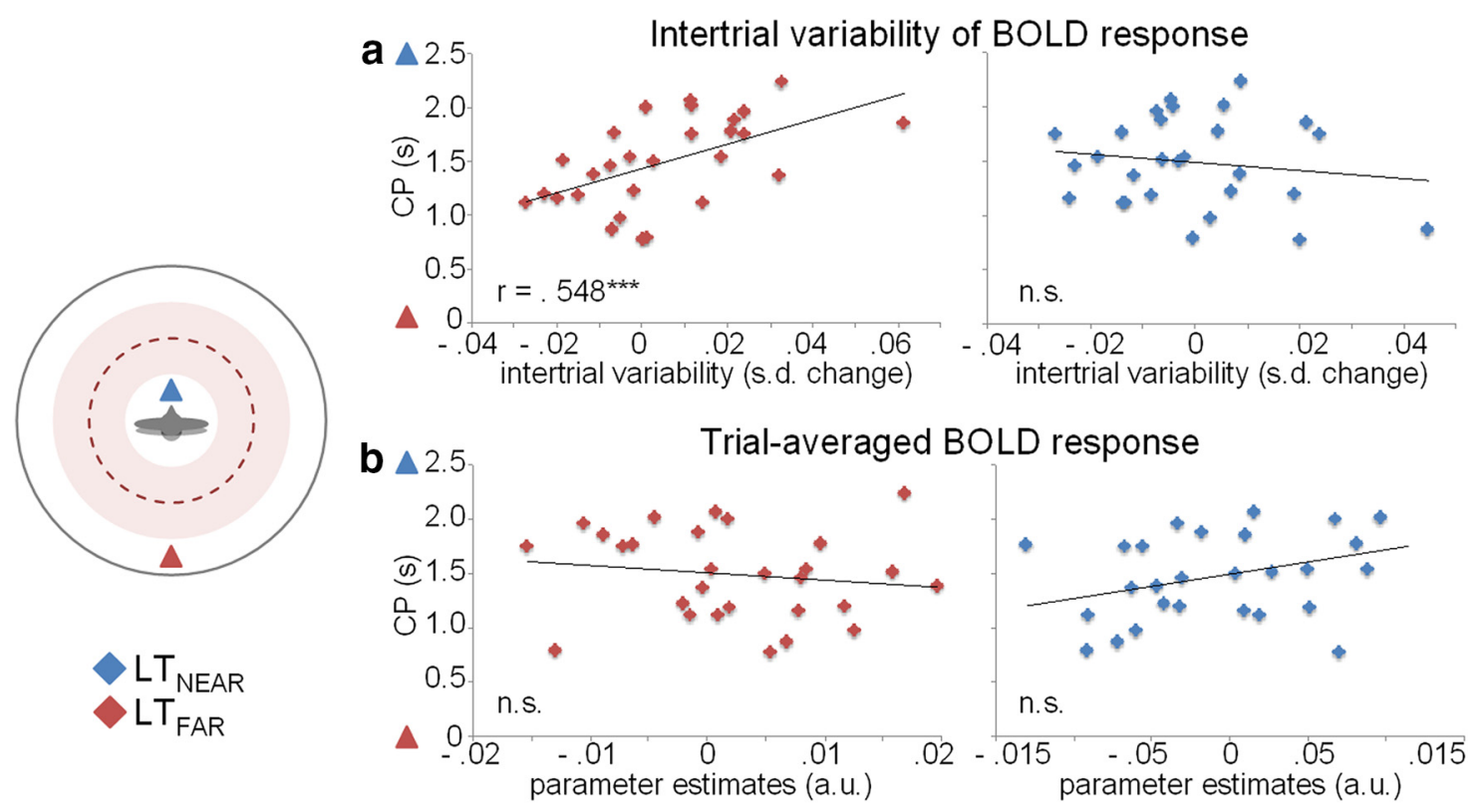

Figure 4. Predictive power of both ITV and trial-averaged BOLD response in PM for individual CPS. Pearson's correlation analyses (bootstrap corrected) of ITV in $L T_{\text {FAR }}$ and $L T_{\text {NEAR }}$ with individual CP values $(\boldsymbol{a})$ and trial-averaged BOLD responses in $L T_{\text {FAR }}$ and $L T_{\text {NEAR }}$ with individual $C P$ values $(\boldsymbol{b})$. Left, Top view of the representation of participants' PPS boundary. The shaded region indicates the total range of $C P$ values across participants, while the dashed red line indicates the group $C P(C P m e a n)$.

However, any conclusion about the convenience of the ITVbased approach rests on its ability to provide information about the individual boundary of PPS that is not provided by the amplitude of averaged BOLD responses. So the question becomes, does trial-averaged amplitude of BOLD responses predict the individual boundary of PPS as much as ITV? To answer this question, we tested whether trial-averaged BOLD responses during either $L T_{\mathrm{FAR}}$ or $L T_{\mathrm{NEAR}}$ conditions predicted the CP. We found no significant correlation in either case $(r=-0.148, p=$ $0.451 ; r=0.401$, bootstrapped $p>0.05$, CI: -0.027 to -0.708 , respectively; Fig. 4).

All in all, these results suggest that ITV of BOLD responses (ITV) in PM to audio-tactile stimuli, rather than trial-averaged amplitude of BOLD responses, contributes to define the individual location of PPS boundary (CP).

\section{Increased ITV in far space in individuals with narrow} PPS boundary

To further investigate the relationship between ITV in PM and the individual differences in PPS, we separated our participants into two groups according to their CP by a median split. This analysis was conducted first to provide further support to the specific relationship between ITV in the far space condition and the CP and second to investigate whether ITV dynamics in PM differentiated between near and far space in individuals with either wide or narrow PPS. One group of participants was characterized by low CP values corresponding to a distant-from-thebody location of PPS boundary, therefore a wide PPS (the CPw group). The other group was characterized by high CP values indicating that their PPS boundary was located near to their body, so a narrow PPS (the $\mathrm{CP} n$ group).

One-sample $t$ test analyses against zero with each ITV time course normalized to the first frame showed increased ITV for the far space condition in the CPn group ( $t_{13}=2.912, p=0.012$, two-tailed), but not in the CP $w$ group $\left(\mathrm{t}_{13}=-1.177, p=0.26\right.$, two-tailed) (Fig. $5 a-c)$. Neither group showed any significant modulation of ITV for the near space condition (CPn group: $t_{13}=-0.51, p=0.618$; $\mathrm{CP} w$ group: $t_{13}=0.057, p=0.955$, two-tailed).
Mixed ANOVA on ITV values showed a significant interaction $\left(F_{(1,26)}=8.024, p=0.009\right)$ between the two factors group $(\mathrm{CP} n, \mathrm{CP} w)$ and space $\left(L T_{\mathrm{NEAR}}, L T_{\mathrm{FAR}}\right)$ (Fig. 6). Simple effect analyses revealed that the $\mathrm{CP} n$ and the $\mathrm{CP} w$ groups differed in the $L T_{\mathrm{FAR}}$ condition $\left(t_{26}=3.014, p=0.006\right)$, but not in the $L T_{\mathrm{NEAR}}$ condition $\left(t_{26}=-0.351, p=0.728\right)$. Moreover, comparisons within each group revealed that ITV significantly differed between $L T_{\mathrm{FAR}}$ and $\mathrm{LT}_{\mathrm{NEAR}}$ conditions only in the $\mathrm{CP} n$ group $(\mathrm{CP} n$ group: $t_{13}=4.272, p<0.001$; $\mathrm{CP} w$ group: $t_{13}=-0.762, p=$ $0.46)$. All in all, these results first support the specific relationship between ITV in the far space condition and CP; indeed, they show a significant difference between the $\mathrm{CP} n$ group and the $\mathrm{CP} w$ group only in the far space condition due to increased variability in the former group. Second, they indicate that a significant increase of the variability in the far space condition with respect to the near space condition allows participants ( $\mathrm{CP} n$ group) to set the boundary of their PPS closer to their body. Conversely, when the variability in the far space is not different from that in the near space, the boundary of participants' PPS extends further (CP $w$ group).

To confirm to a greater extent that the main contribution to individual CPs is provided by ITV rather than trial-averaged amplitude of BOLD responses, we conducted the same analyses on the latter. In this case, results from simple-effect analyses testing between-group differences were not significant.

\section{Discussion}

From where do individual differences in the location of the PPS boundary (Longo and Lourenco, 2007; Lourenco et al., 2011; Sambo and Iannetti, 2013; Taffou and Viaud-Delmon, 2014) originate? We found that they are predicted by the ITV of BOLD response to far stimuli in the PM. Conversely, the trial-averaged amplitude of the same BOLD response did not show any predictive power for behavioral individual differences. How do we interpret this finding? The implicit but key assumption behind any modulation of ITV is that brain responses to a stimulus are embedded in a constantly changing, ongoing activity (He, 2013). 


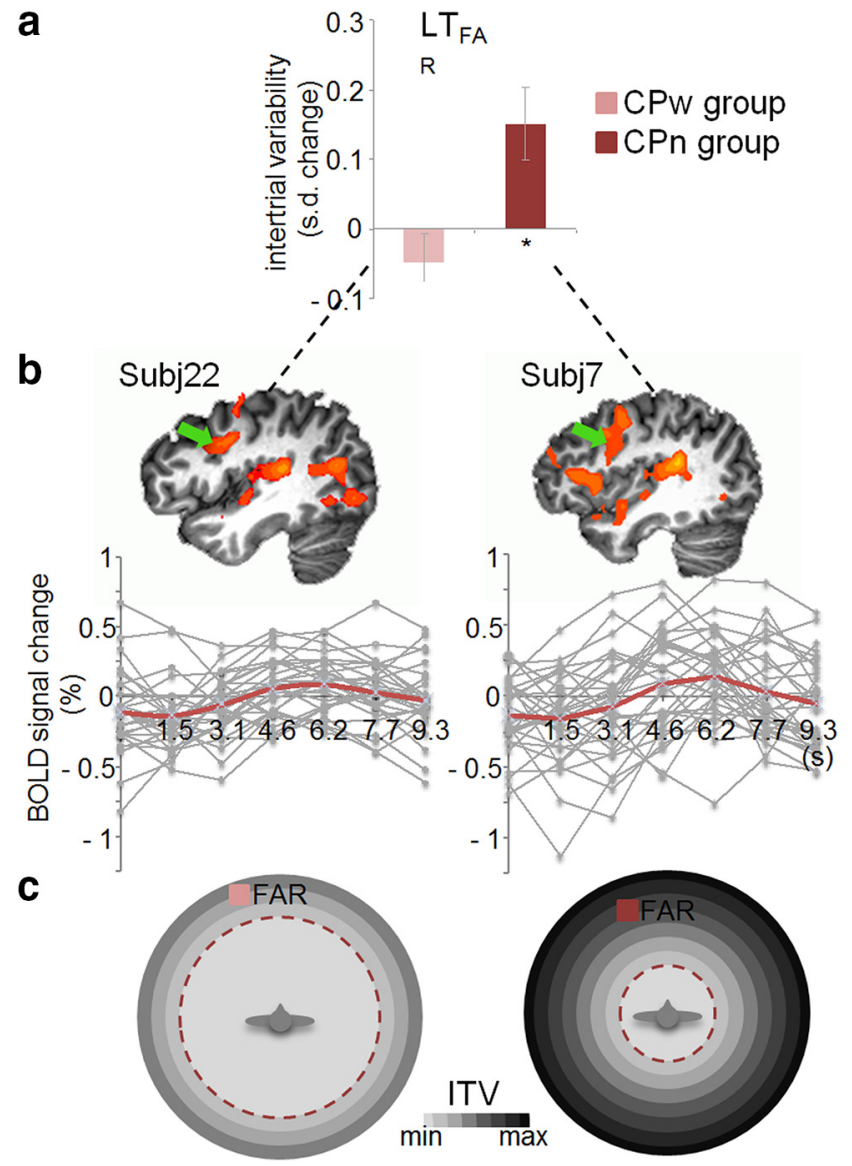

Figure 5. Participants with narrow PPS boundary show increased ITV of BOLD response to far stimuli. (Pw group are participants with low CP values corresponding to a distant-from-thebody location of PPS boundary (i.e., a wide PPS). (Pn group are participants with high CP values corresponding to a near-to-the-the-body location of PPS boundary (i.e., a narrow PPS). $\boldsymbol{a}$, One-sample $t$ test analyses to assess ITV changes in $L T_{\mathrm{FAR}}$ condition in each group (Bonferroni's correction for multiple comparisons). ${ }^{* *} p \leq 0.01$. $\boldsymbol{b}$, Illustration of response variability to $L T_{\text {FAR }}$ condition for a single representative subject from the (Pw group (Subj22) and the CPn group (Subj7). Top, Statistical parametric maps showing voxels significantly activated during $L T_{\text {FAR }}$ condition ( $p>0.005$, corrected). Bottom, Event-related responses in PM for $L T_{\mathrm{FAR}}$ condition. The thick red line in each graph represents the averaged response across the single trials (gray lines) for all time points (gray dots). c, Top views of Subj22's (left) and Subj7's (right) PPS boundary (red dashed lines). Gradients of gray level intensities represent the differences in ITV between the two representative subjects in the far space condition as inferred by the authors.

Each time the same stimulus is presented, the brain responds uniquely depending on its initial condition or the ongoing activity level at stimulus onset (Fox et al., 2006; He, 2013). Therefore, brain responses to the same stimulus are variable and such variability may carry information that is lost after averaging (Garrett et al., 2010). ITV dynamics, in particular, can capture this information because they are calculated in reference to fluctuations at stimulus onset (He, 2013). In our study, the predictive power of ITV for individual PPS boundary would thus imply a relationship between constantly changing ongoing activity in PM and the individual PPS boundary. In other words, the location of the safety margin around the body would not simply rely upon how much a participant reacts to a particular environmental stimulus; rather, it would reflect the range of ongoing activity-dependent variability of his/her responses to that stimulus. Specifically, we found increased ITV in premotor responses to stimuli in the far space only in participants with a narrow PPS boundary. This suggests that individual differences in PPS extension derive from
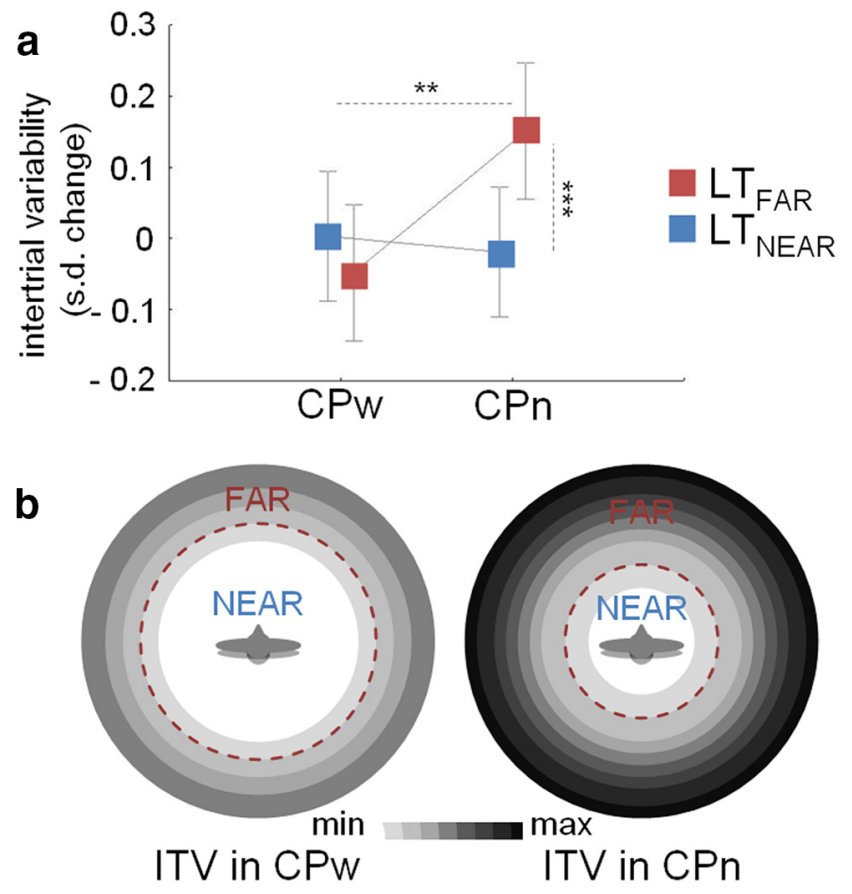

Figure 6. Participants with different extension of PPS show different modulation of ITV in the near and far conditions. $\boldsymbol{a}$, Significant $\left(F_{(1,34)}=9.208, p=0.005\right)$ interaction of group $(C P n, C P W) \times$ space $\left(L T_{\text {NEAR, }}, L T_{\text {FAR }}\right)$, and the results from simple effect analyses (Bonferroni correction). ${ }^{*} p<0.05 ;{ }^{* *} p=0.001$. $\boldsymbol{b}$, Top views of PPS boundaries (red dashed lines) and gradients of ITV (different gray level intensities) in the CPw group (left) and the CPn group (right). Note that gradients of ITV correspond to the authors' interpretation of the data.

ongoing activity-dependent variability of premotor responses to far and unreliable (because of the low intensity of the sound), rather than near and reliable (because of the high intensity of the sound), stimuli.

If the hypothesis of a link between brain ongoing activity and individual PPS boundary is at stake, it would have interesting implications for understanding symptoms of altered PPS boundary in psychiatric disorders, such as in anxiety (Sambo and Iannetti, 2013) and schizophrenia (Park et al., 2009), both characterized by increased safety margin surrounding the body.

One question as to whether such variability might be advantageous for adaptive changes of individual PPS extension may arise at this point. How much do our findings align with the well known plasticity and dynamic modulation of PPS extension induced, for example, in different contexts (Tajadura-Jiménez et al., 2010; Teneggi et al., 2013)? In general, variability may allow the brain to operate in a Bayesian manner (Knill and Pouget, 2004): it estimates the reliability/uncertainty of incoming stimuli and optimally chooses from a range of options. Specifically, brain variability would increase upon greater stimulus uncertainty (far objects) relative to conditions of less stimulus uncertainty (near objects). However, this transition may vary across individuals as a function of, for example, prior experience and individual skills. There is already empirical evidence suggesting that the transition from higher to lower variability of brain responses may be more subtle in older, poorer performing adults due to reduced neural flexibility (Garrett et al., 2013a).

According to the Bayesian view, variability would be beneficial while facing moment-to-moment changes in stimulus information and adapt across levels of uncertainty in the external world. Conversely, if the brain responded in the same manner each time a certain stimulus was encountered, then there would be less chance to 
cope with different circumstances involving that stimulus. For example, an approaching object at a specific distance from the body may be threatening, such as a dangerous animal, or not.

Neurophysiological mechanisms of multisensory PPS coding PPS representation is enabled through the integration of acoustic (or visual) information emanating from events occurring in the vicinity of the body and somatosensory information originating on the same body (Macaluso and Maravita, 2010; Occelli et al., 2011). Audio-tactile PPS neurons have been described previously (Graziano et al., 1999) in monkey PM. They show a gradient of firing that varies as a function of distance. Analogous forms of multisensory responses have been described also in humans (Bremmer et al., 2001; Makin et al., 2007; Serino et al., 2011). How could the properties of multisensory PPS neurons help us to understand the increase of ITV in the far space condition?

A recent PPS computational model (Serino et al., 2015) can provide hints to answer this question. Using the same audiotactile paradigm as in the present study, the investigators showed that the model effectively reproduced the characteristic behavior of multisensory PPS neurons. Briefly, the model includes two unisensory areas (tactile and auditory) communicating with a third multisensory area (audio-tactile) via synaptic connections. Unimodal stimulation, either a tactile stimulus on the hand or an auditory stimulus in the near space, activates the corresponding unisensory area as well as the multisensory neuron. On the contrary, an auditory stimulus from the far space activates the unisensory neurons, but only weakly stimulates the multisensory ones, if any.

How could such behavior of multisensory PPS neurons affect ITV of neural response? It is possible to hypothesize that, when a far and unreliable auditory stimulus weakly activates only a few PM neurons, it generates a highly variable unisensory response (Sarko et al., 2013). The probability for a tactile stimulus occurring in such a context to generate a multisensory response in PM neurons is low, but likely injects more variability into their response at each trial. As a result, ITV should increase. Conversely, when a near and reliable auditory stimulus activates most of the PM neurons, the variability of the unisensory response is low. The probability for a tactile stimulus to generate a multisensory response in this context is high at each trial. As a result, ITV in PM should not increase appreciably.

According to this hypothesis, we propose to conceive the space around the body as a gradient of response variability (Figs. $5 c, 6 b)$ originating from the likelihood for multisensory responses to occur in time and space. PPS would correspond to the space around the body characterized by low response variability and high probability for multisensory responses to occur due to high reliability of external cues. According to our results, such low response variability within PPS is a common feature across individuals. Conversely, the far space would be characterized by increased response variability (potentiality for adaptation and plasticity) and low probability for multisensory responses to occur due to the low reliability of external cues. However, as suggested by our results, it is such increased variability that changes across individuals and predicts the individual location of PPS boundary. To unambiguously support the hypothesis of the space around the body as a gradient of response variability (Figs. $5 c, 6 b)$ to multisensory stimuli, future studies should quantify ITV associated with several points around the individual CP.

If our argument is at stake, one may formulate interesting hypotheses on how the extension of PPS, such as after tool use (Farnè et al., 2005; Vaesen, 2012; Cléry et., 2015), may be related to neural variability of premotor responses to far stimuli. This approach would also provide a causal, rather than merely correlational, connection for the link between ITV and individual extension of PPS. Another possible approach could be to modulate the motor coding of PPS using TMS (Avenanti et al., 2012) and see how this is related to ITV changes in the PM.

It may be surprising that, unlike previous neuroimaging studies (Makin et al., 2007; Brozzoli et al., 2011, 2013), we did not find intraparietal regions to be involved in coding PPS. One possible explanation is that prior studies used visuo-tactile, rather than audio-tactile, interaction paradigms, whereas it is known that the auditory modality is much less represented than the visual modality in the intraparietal cortex (Guipponi et al., 2013). Moreover, intraparietal regions do not seem to be sensitive to increasing sound intensity. In this respect, our data confirmed previous evidence by Seifritz et al. (2002), who did not observe specific activity for looming sounds in parietal areas more superior to the temporoparietal junction. Finally, results from the whole-brain analysis (Fig. $3 d$ ) rule out the possibility that a restrictive selection of the ROIs prevented us from finding any involvement of the posterior parietal cortex in coding PPS, as elicited by looming sounds.

\section{Conclusions}

In sum, we have shown that individual differences of the PPS boundary are specifically predicted by variability of BOLD responses in the PM to far stimuli approaching our body. We propose that increased variability of premotor responses observed in individuals with narrow PPS may provide them with a margin for dynamic and successful adaption to events occurring in the external world. Our results provide the first empirical support for the relevance of regional ITV for human behavior and its variance across individuals.

\section{References}

Avenanti A, Annela L, Serino A (2012) Suppression of premotor cortex disrupts motor coding of peripersonal space. Neuroimage 63:281-288. CrossRef Medline

Bremmer F, Schlack A, Shah NJ, Zafiris O, Kubischik M, Hoffmann K, Zilles K, Fink GR (2001) Polymodal motion processing in posterior parietal and premotor cortex: a human fMRI study strongly implies equivalencies between humans and monkeys. Neuron 29:287-296. CrossRef Medline

Brozzoli C, Gentile G, Petkova VI, Ehrsson HH (2011) FMRI adaptation reveals a cortical mechanism for the coding of space near the hand. J Neurosci 31:9023-9031. CrossRef Medline

Brozzoli C, Gentile G, Bergouignan L, Ehrsson HH (2013) A shared representation of the space near oneself and others in the human premotor cortex. Curr Biol 23:1764-1768. CrossRef Medline

Brozzoli C, Ehrsson HH, Farnè A (2014) Multisensory representation of the space near the hand: from perception to action and interindividual interactions. Neuroscientist 20:122-135. CrossRef Medline

Canzoneri E, Magosso E, Serino A (2012) Dynamic sounds capture the boundaries of peripersonal space representation in humans. PLoS One 7:e44306. CrossRef Medline

Carandini M (2004) Amplification of trial-to-trial response variability by neurons in visual cortex. PLoS Biol 2:E264. CrossRef Medline

Churchland MM, Yu BM, Ryu SI, Santhanam G, Shenoy KV (2006) Neural variability in premotor cortex provides a signature of motor preparation. J Neurosci 26:3697-3712. CrossRef Medline

Cléry J, Guipponi O, Wardak C, Ben Hamed S (2015) Neuronal bases of peripersonal and extrapersonal spaces, their plasticity and their dynamics: knowns and unknowns. Neuropsychologia 70:313-326. Medline

Colby CL, Duhamel JR, Goldberg ME (1993) Ventral intraparietal area of the macaque: anatomic location and visual response properties. J Neurophysiol 69:902-914. Medline

Cox RW (1996) AFNI: software for analysis and visualization of functional magnetic resonance neuroimages. Comput Biomed Res 29:162-173. CrossRef Medline 
Faisal AA, Selen LP, Wolpert DM (2008) Noise in the nervous system. Nat Rev Neurosci 9:292-303. CrossRef Medline

Farnè A, Iriki A, Làdavas E (2005) Shaping multisensory action-space with tools: evidence from patients with cross-modal extinction. Neuropsychologia 43:238-248. CrossRef Medline

Ferri F, Tajadura-Jiménez A, Väljamäe A, Vastano R, Costantini M (2015) Emotion-inducing approaching sounds shape the boundaries of multisensory peripersonal space. Neuropsychologia 70:468-475. CrossRef Medline

Fogassi L, Gallese V, Fadiga L, Luppino G, Matelli M, Rizzolatti G (1996) Coding of peripersonal space in inferior premotor cortex (area F4). J Neurophysiol 76:141-157. Medline

Fotowat H, Gabbiani F (2011) Collision detection as a model for sensorymotor integration. Annu Rev Neurosci 34:1-19. CrossRef Medline

Fox MD, Snyder AZ, Zacks JM, Raichle ME (2006) Coherent spontaneous activity accounts for trial-to-trial variability in human evoked brain responses. Nat Neurosci 9:23-25. CrossRef Medline

Fox MD, Snyder AZ, Vincent JL, Raichle ME (2007) Intrinsic fluctuations within cortical systems account for intertrial variability in human behavior. Neuron 56:171-184. CrossRef Medline

Garrett DD, Kovacevic N, McIntosh AR, Grady CL (2010) Blood oxygen level-dependent signal variability is more than just noise. J Neurosci 30: 4914-4921. CrossRef Medline

Garrett DD, Kovacevic N, McIntosh AR, Grady CL (2013a) The modulation of BOLD variability between cognitive states varies by age and processing speed. Cereb Cortex 23:684-693. CrossRef Medline

Garrett DD, Samanez-Larkin GR, MacDonald SW, Lindenberger U, McIntosh AR, Grady CL (2013b) Moment-to-moment brain signal variability: a next frontier in human brain mapping? Neurosci Biobehav Rev 37: 610-624. CrossRef Medline

Graziano MS, Cooke DF (2006) Parieto-frontal interactions, personal space, and defensive behavior. Neuropsychologia 44:845-859. CrossRef Medline

Graziano MS, Hu XT, Gross CG (1997) Visuospatial properties of ventral premotor cortex. J Neurophysiol 77:2268-2292. Medline

Graziano MS, Reiss LA, Gross CG (1999) A neuronal representation of the location of nearby sounds. Nature 397:428-430. CrossRef Medline

Guipponi O, Wardak C, Ibarrola D, Comte JC, Sappey-Marinier D, Pinède S, Ben Hamed S (2013) Multimodal convergence within the intraparietal sulcus of the macaque monkey. J Neurosci 33:4128-4139. CrossRef Medline

He BJ (2013) Spontaneous and task-evoked brain activity negatively interact. J Neurosci 33:4672-4682. CrossRef Medline

James TW, Stevenson RA, Kim S (2012) Inverse effectiveness and BOLD fMRI. In: The new handbook of multisensory processes (Stein BE, ed.), pp 207-222. Cambridge, MA: MIT.

Kanai R, Rees G (2011) The structural basis of inter-individual differences in human behaviour and cognition. Nat Rev Neurosci 12:231-242. Medline

Kim S, Stevenson RA, James TW (2012) Visuo-haptic neuronal convergence demonstrated with an inversely effective pattern of BOLD activation. J Cogn Neurosci 24:830-842. CrossRef Medline

Knill DC, Pouget A (2004) The Bayesian brain: the role of uncertainty in neural coding and computation. Trends Neurosci 27:712-719. CrossRef Medline

Kriegeskorte N, Simmons WK, Bellgowan PS, Baker CI (2009) Circular analysis in systems neuroscience: the dangers of double dipping. Nat Neurosci 12:535-540. CrossRef Medline

Làdavas E, Serino A (2008) Action-dependent plasticity in peripersonal space representations. Cogn Neuropsychol 25:1099-1113.

Longo MR, Lourenco SF (2007) Space perception and body morphology: extent of near space scales with arm length. Exp Brain Res 177:285-290. CrossRef Medline

Lourenco SF, Longo MR, Pathman T (2011) Near space and its relation to claustrophobic fear. Cognition 119:448-453. CrossRef Medline

Macaluso E, Maravita A (2010) The representation of space near the body through touch and vision. Neuropsychologia 48:782-795. CrossRef Medline

Makin TR, Holmes NP, Zohary E (2007) Is that near my hand? Multisensory representation of peripersonal space in human intraparietal sulcus. J Neurosci 27:731-740. CrossRef Medline

Mandelblat-Cerf Y, Paz R, Vaadia E (2009) Trial-to-trial variability of single cells in motor cortices is dynamically modified during visuomotor adaptation. J Neurosci 29:15053-15062. CrossRef Medline

Maravita A, Spence C, Driver J (2003) Multisensory integration and the body schema: close to hand and within reach. Curr Biol 13:R531-R539. CrossRef Medline

Marcos E, Pani P, Brunamonti E, Deco G, Ferraina S, Verschure P (2013) Neural variability in premotor cortex is modulated by trial history and predicts behavioral performance. Neuron 78:249-255. CrossRef Medline

Noppeney U (2012) Characterization of multisensory integration with fMRI: experimental design, statistical analysis, and interpretation. In: The neural bases of multisensory processes (Murray MM, Wallace MT, eds.). Boca Raton, FL: CRC.

Occelli V, Spence C, Zampini M (2011) Audiotactile interactions in front and rear space. Neurosci Biobehav Rev 35:589-598. CrossRef Medline

Park SH, Ku J, Kim JJ, Jang HJ, Kim SY, Kim SH, Kim CH, Lee H, Kim IY, Kim SI (2009) Increased personal space of patients with schizophrenia in a virtual social environment. Psychiatry Res 169:197-202. CrossRef Medline

Rizzolatti G, Scandolara C, Matelli M, Gentilucci M (1981) Afferent properties of periarcuate neurons in macaque monkeys. II. Visual responses. Behav Brain Res 2:147-163. CrossRef Medline

Rizzolatti G, Fadiga L, Fogassi L, Gallese V (1997) The space around us. Science 277:190-191. CrossRef Medline

Rokni U, Richardson AG, Bizzi E, Seung HS (2007) Motor learning with unstable neural representations. Neuron 54:653-666. CrossRef Medline

Sambo CF, Iannetti GD (2013) Better safe than sorry? The safety margin surrounding the body is increased by anxiety. J Neurosci 33:14225-14230. CrossRef Medline

Sarko DK, Ghose D, Wallace MT (2013) Convergent approaches toward the study of multisensory perception. Front Syst Neurosci 7:81. Medline

Scaglione A, Moxon KA, Aguilar J, Foffani G (2011) Trial-to-trial variability in the responses of neurons carries information about stimulus location in the rat whisker thalamus. Proc Natl Acad Sci U S A 108:14956-14961. CrossRef Medline

Schiff W (1965) Perception of impending collision: a study of visually directed avoidant behavior. Psychol Monogr 79:11:11-26. Medline

Seifritz E, Neuhoff JG, Bilecen D, Scheffler K, Mustovic H, Schächinger H, Elefante R, Di Salle F (2002) Neural processing of auditory looming in the human brain. Curr Biol 12:2147-2151. CrossRef Medline

Serino A, Canzoneri E, Avenanti A (2011) Fronto-parietal areas necessary for a multisensory representation of peripersonal space in humans: an rTMS study. J Cogn Neurosci 23:2956-2967. CrossRef Medline

Serino A, Canzoneri E, Marzolla M, di Pellegrino G, Magosso E (2015) Extending peripersonal space representation without tool-use: evidence from a combined behavioral-computational approach. Front Behav Neurosci 9:4. Medline

Taffou M, Viaud-Delmon I (2014) Cynophobic fear adaptively extends peri-personal space. Front Psychiatry 5:122. Medline

Tajadura-Jiménez A, Larsson P, Väljamäe A, Västfjäll D, Kleiner M (2010) When room size matters: acoustic influences on emotional responses to sounds. Emotion 10:416-422. CrossRef Medline

Talairach J, Tournoux P (1988) Co-planar stereotaxic atlas of the human brain: 3-D proportional system: an approach to cerebral imaging. New York: Thieme.

Teneggi C, Canzoneri E, di Pellegrino G, Serino A (2013) Social modulation of peripersonal space boundaries. Curr Biol 23:406-411. CrossRef Medline

Vaesen K (2012) The cognitive bases of human tool use. Behav Brain Sci 35:203-218. CrossRef Medline

Werner S, Noppeney U (2010) Superadditive responses in superior temporal sulcus predict audiovisual benefits in object categorization. Cereb Cortex 20:1829-1842. CrossRef Medline

Xiong J, Gao J, Lancaster J, Fox P (1995) Clustered pixels analysis for functional MRI activation studies of the human brain. Hum Brain Mapp 3:287-301. CrossRef

Zilles K, Amunts K (2013) Individual variability is not noise. Trends Cogn Sci 17:153-155. CrossRef Medline 